\title{
Análise de indicadores epidemiológicos de crianças e adolescentes acometidos pela Covid-19 no Nordeste do Brasil
}

Analysis of epidemiological indicators of children and adolescents affected by Covid-19 in Northeastern Brazil

Análisis de indicadores epidemiológicos de niños y adolescentes afectados por Covid-19 en el Noreste de Brasil

\section{João Cruz Neto', Emanuel Messias Silva Feitosa ${ }^{\text {II }}$, Kely Vanessa Leite Gomes da Silva ${ }^{\text {III, }}$ Célida Juliana de Oliveira ${ }^{\mathrm{IV}}$}

\begin{abstract}
Resumo: Objetivo: analisar os indicadores epidemiológicos de crianças e adolescentes acometidos pela Covid-19 na região Nordeste do Brasil. Método: estudo ecológico com casos de Covid-19, nos estados do Nordeste. Calculouse taxas de letalidade, prevalência, índice acumulado diário e razão de leitos de UTI. Analisou-se dados consolidados até 21 de agosto de 2020. Resultados: houve 74.705 casos de Covid-19, com prevalência do sexo feminino e quanto a faixa etária àqueles entre 10 a 19 anos. Letalidade foi de 8\%, prevalência de 321/100.000 habitantes e razão de leitos menor que dois. Houve significância para relação entre o maior número de óbitos e o menor número de leitos de UTI ( $\mathrm{p}=0.001$ ), maior prevalência de casos em razão da menor quantidade de leitos de UTI ( $\mathrm{p}=0.007$ ). Conclusão: os indicadores revelam insuficiência quanto aos leitos para a doença, alta letalidade e prevalência, bem como quantidade razoável dos leitos de UTI.

Descritores: Infecções por coronavírus; Indicadores básicos de saúde; Saúde da criança; Saúde do adolescente; Epidemiologia
\end{abstract}

\begin{abstract}
Objective: to analyze the epidemiological indicators of children and adolescents affected by Covid-19 in the Northeast region of Brazil. Method: ecological study with cases of Covid-19, in the northeastern states. Lethality, prevalence, daily accumulated index and ICU bed ratio were calculated. Consolidated data were analyzed until August 21, 2020. Results: there were 74,705 cases of Covid-19, with female prevalence and age group between 10 and 19 years. Lethality was $8 \%$, prevalence of 321/100,000 inhabitants and bed ratio lower than two. There was
\end{abstract}

\footnotetext{
I Acadêmico de Enfermagem, Universidade Regional do Cariri (URCA), Crato, Ceará, Brasil. E-mail: enfjcncruz@gmail.com, Orcid: https://orcid.org/ 0000-0002-0972-2988.

II Acadêmico de Enfermagem, Universidade Regional do Cariri (URCA), Crato, Ceará, Brasil. E-mail: emfeitosa2017@gmail.com, Orcid: https://orcid.org/0000-0001-5278-3105.

III Enfermeira, Doutora em Enfermagem, Universidade Regional do Cariri (URCA)/Docente do departamento de Enfermagem da Universidade Regional do Cariri, Crato, Ceará, Brasil. E-mail: kelyvanessa@hotmail.com, Orcid: https://orcid.org/0000-0001-5179-8962.

IV Enfermeira, Doutora em Enfermagem, Universidade Regional do Cariri (URCA)/Docente do departamento de Enfermagem da Universidade Regional do Cariri, Crato, Ceará, Brasil. E-mail: celida.oliveira@urca.br, Orcid: https://orcid.org/0000-0002-8900-6833.
} 
Análise de indicadores epidemiológicos de crianças e adolescentes acometidos pela Covid... $\mid 2$

significance for the relationship between the highest number of deaths and the lowest number of ICU beds $(\mathrm{p}=0.001)$, higher prevalence of cases due to the lower number of ICU beds $(\mathrm{p}=0.007)$. Conclusion: the indicators reveal insufficiency regarding beds for the disease, high lethality and prevalence, as well as a reasonable amount of ICU beds.

Descriptors: Coronavirus Infections; Health Status Indicators; Child Health; Adolescent Health; Epidemiology

Resumen: Objetivo: analizar los indicadores epidemiológicos de los niños y adolescentes afectados por Covid-19 en la región noreste de Brasil. Método: estudio ecológico con casos de Covid-19, en los estados del noreste. Se calcularon la letalidad, la prevalencia, el índice acumulado diario y la proporción de lechos de la UCI. Los datos consolidados se analizaron hasta el 21 de agosto de 2020. Resultados: hubo 74.705 casos de Covid-19, con prevalencia femenina y grupo de edad entre 10 y 19 años. La letalidad fue del 8\%, la prevalencia de 321/100.000 habitantes y la proporción de camas inferior a dos. Hubo importancia para la relación entre el mayor número de muertes y el menor número de camas de UCI ( $\mathrm{p}=0,001)$, mayor prevalencia de casos debido al menor número de camas de UCI $(\mathrm{p}=0,07)$. Conclusión: los indicadores revelan insuficiencia con respecto a las camas para la enfermedad, alta letalidad y prevalencia, así como una cantidad razonable de camas de UCI.

Descriptores: Infecciones por Coronavirus; Indicadores de salud; Salud del niño; Salud del adolescente; Epidemiología

\section{Introdução}

A pandemia da coronavirus infectious disease (Covid-19) se configura como uma ameaça global e, segundo dados da World Health Organization, já são 61.299.371 casos confirmados, incluindo 1.439.784 mortos até a data de 28 de novembro de 2020. No Brasil, acumulam-se 6.238.35 casos confirmados, com 171.974 óbitos. $^{1}$

Dada a importância ao contexto da pandemia da Covid-19, precisa-se considerar vários aspectos relacionados à saúde. As implicações do severe acute respiratory syndrome coronavirus 2 (SARS-CoV-2) são amplas e várias faixas etárias têm sido acometidas, dentre elas, crianças e adolescentes, que se configuram por serem períodos de idade vulneráveis a diversos problemas de saúde. ${ }^{2}$ De acordo com uma projeção realizada na Itália em crianças e adolescentes, duas em cada dez têm teste positivo para Sars-Cov-2 ou possuem a síndrome respiratória aguda grave. ${ }^{3}$

Em Wuhan, na província de Hubei na China, das 1391 crianças que foram testadas até março de 2020, 171 (12,3\%) deram positivo para o Sars-Cov-2, com média de até sete anos, sendo elas um dos principiais meios de disseminação do vírus, principalmente pelas características 
assintomáticas. Destas, $27(15,8 \%)$ apresentaram alterações com opacidade bilateral ao exame radiológico, indicando pneumonia e linfopenia persistentes. ${ }^{4}$

Estudo realizado nos Estados Unidos da América com 192 crianças e adolescentes, que objetivou alertar sobre o plano de retomada das escolas norte-americanas e a possível disseminação em massa do vírus, observou que $26 \%$ das crianças estavam infectadas pelo SarsCov-2 e outros $8 \%$ com critérios precisos da síndrome inflamatória multissistêmica. Destas, apenas 25 apresentaram febre como sintoma não específico, além de tosse, congestão, rinorreia e dor de cabeça, o que pode levar a uma alta na transmissão e internação hospitalar significativa de adultos. ${ }^{5}$

Os casos de Covid-19 em crianças ainda são discretos, de maioria assintomática e com fatores desconhecidos, quando se associam a comorbidades adjacentes, como as doenças do aparelho respiratório, ocasionando maior taxa de morbimortalidade. ${ }^{6} \mathrm{Em}$ pacientes pediátricos, há uma rápida infecção e disseminação da doença com acréscimo gradual na curva de infectados. $^{7}$

Existem relativamente poucos casos relatados de infecção causada por Covid-19 em crianças em comparação com o número total de casos na população em geral. Dados da direção geral de saúde de Portugal, de 29 de março de 2020, demonstraram que 1\% das crianças menores de 10 anos e 2,3\% dos adolescentes de 11 a 19 anos estavam apresentando a Covid-19. ${ }^{8}$ Apesar da subnotificação, até 16 de junho de 2020, foram comunicados mundialmente mais de 8 milhões de casos em crianças e adolescentes confirmados para Covid-19, sendo que os casos graves requerem suporte ventilatório por tempo prolongado. ${ }^{1,9}$

O espectro clínico da infecção pelo Sars-Cov-2 é amplo, mas, basicamente, pode se apresentar em três principais condições: portadores assintomáticos, indivíduos com doença respiratória aguda (DRA) ou pacientes com pneumonia em diferentes graus de gravidade. Estas 
Análise de indicadores epidemiológicos de crianças e adolescentes acometidos pela Covid... | 4

duas últimas condições são consideradas como agravos de saúde relacionados à infecção pelo Sars-Cov-2. ${ }^{10-11}$

Embora o risco imediato da Covid-19 em crianças seja baixo, é importante acompanhar a situação e sua evolução. Apesar de notícias não graves em crianças, um estudo com 171 crianças revelou que durante a hospitalização, apenas três necessitaram de suporte intensivo e ventilação mecânica invasiva, sendo que todos tinham condições coexistentes como hidronefrose, leucemia, intussuscepção e linfopenia. Nesses casos, houve morte devido a falência múltipla de órgãos. ${ }^{4,8}$

O número de casos notificados da Covid-19 certamente subestima a verdadeira carga da doença, devido à indisponibilidade de testes em vários locais e também devido à proporção de pessoas que desenvolvem formas assintomáticas ou leves não identificadas da doença, apesar de estarem infectadas..$^{12}$ A letalidade do Sars-Cov-2 varia, principalmente, conforme a faixa etária e as condições clínicas dos indivíduos acometidos. ${ }^{13}$

$\mathrm{Na}$ região Nordeste do Brasil, os casos de crianças e adolescentes com Covid-19 destacam-se em relação à realidade nacional, especialmente pelas vulnerabilidades existentes. Há desigualdades marcantes como o acesso à água encanada, esgoto sanitário e energia elétrica com quase $10 \%$ dos domicílios abaixo da linha da pobreza com fragilidades especialmente na saúde. $^{14}$ Nesse sentido, o presente estudo teve como objetivo analisar os indicadores epidemiológicos de crianças e adolescentes acometidas pela Covid-19 na região Nordeste do Brasil.

\section{Método}

Trata-se de um estudo ecológico do tipo descritivo, cujos locais de pesquisa foram os nove estados da região Nordeste brasileira: Maranhão, Piauí, Ceará, Rio Grande do Norte, Paraíba, Pernambuco, Alagoas, Sergipe e Bahia. 
5 | Neto JC, Feitosa EMS, Silva KVLG, Oliveira CJ

De acordo com a projeção do Instituto Brasileiro de Geografia e Estatística (IBGE) a região nordeste conta com cerca de 57.071.654 milhões de habitantes e uma área territorial de 1.554.257 $\mathrm{km}^{2}$. Dentre os estados com maior número de crianças e adolescentes está a Bahia (9.917.375), Ceará (3.035.091) e o Pernambuco (2.664.267). ${ }^{15}$ A população do estudo foi composta por 23.835.722 crianças e adolescentes. Foram incluídas crianças de 0 a 9 anos e adolescentes de 10 a 19 anos notificados com Covid-19 pelos boletins epidemiológicos dos estados estudados, excluindo todos aqueles maiores desta faixa etária.

Na região Nordeste, os hospitais que tinham 99 leitos sofreram redução de até 6\% na taxa de ocupação. Assim também, as unidades de terapia intensiva (UTI) neonatais reduziram em 5\% sua capacidade de ocupação. Quanto à esfera jurídica, houve aumento das instituições públicas administradas pelo sistema único de saúde (SUS) e diminuição das entidades empresariais e sem fins lucrativos de até $11 \% .^{16}$

A coleta dos dados foi realizada por dois pesquisadores distintos com posterior dupla checagem da amostra em estudo, permitindo a comparação dos achados e minimização de perdas. A pesquisa selecionou os dados consolidados até a $34^{\mathrm{a}}$ semana epidemiológica por meio dos dados disponibilizados pelas secretarias estaduais de saúde em seus endereços eletrônicos (websites) oficiais, conforme o Quadro 1:

Quadro 1- Endereços eletrônicos dos estados analisados, Brasil, 2020.

\begin{tabular}{|l|l|}
\hline \multicolumn{1}{|c|}{ Estado } & \multicolumn{1}{c|}{ Website } \\
\hline Maranhão & https:/www.saude.ma.gov.br/ \\
\hline Ceará & https://integrasus.saude.ce.gov.br/ \\
\hline Rio Grande do Norte & https://portalcovid19.saude.rn.gov.br/ \\
\hline Piauí & http://www.saude.pi.gov.br/ \\
\hline Paraíba & https://paraiba.pb.gov.br/diretas/saude/coronavirus \\
\hline Pernambuco & https://www.cievspe.com/novo-coronavirus-2019-ncov \\
\hline Alagoas & http://www.alagoascontraocoronavirus.al.gov.br/ \\
\hline Sergipe & https://todoscontraocorona.net.br/ \\
\hline Bahia & http://www.saude.ba.gov.br/temasdesaude/coronavirus/ \\
\hline
\end{tabular}


Análise de indicadores epidemiológicos de crianças e adolescentes acometidos pela Covid... | 6

Dados sobre a capacidade dos leitos hospitalares, incluindo UTI neonatais e pediátricas, foram obtidos no cadastro nacional de estabelecimentos de saúde (CNES). Os dados sobre a população geral foram obtidos pelo sítio eletrônico do IBGE e pelo departamento de tecnologias do SUS (DATASUS) com as características populacionais do censo nacional de 2010 e suas estimativas para o ano de 2020.

As variáveis analisadas foram número de casos confirmados, número de óbitos, número de leitos hospitalares e o número de leitos de UTI. Foram considerados os pacientes com resultado laboratorial positivo para o vírus da Covid-19, independentemente de sinais ou sintomas. Além disso, obteve-se o sexo, idade e faixa etária ( 0 a 19 anos).

A partir do número absoluto de casos e óbitos por Covid-19, extraídos pelo método de análise do Sistema de Informação em Saúde do qual os dados são provenientes, foram calculadas as taxas que se seguem: prevalência (nº de caso confirmados/população residente x 100.000hab), letalidade (no de óbitos por Covid-19/total de casos confirmados x 100) e o índice acumulado diário (IAD) (nº de casos confirmados/nº de dias entre o primeiro caso relatado e 21 de agosto de 2020).

A análise foi realizada mediante os cálculos para capacidade de leitos hospitalares, a frequência absoluta e relativa e os valores mínimo e máximo para o número de casos com auxílio do programa Microsoft Excel for Windows 2016 ${ }^{\circledR}$. Os dados foram submetidos ao teste de normalidade Shapiro-Wilk, com posterior análise estatística paramétrica com o teste $t$ de Student, pelo Software R versão 4.0.2 for Windows ${ }^{\circ}$. Em todos os casos foram utilizados os critérios de significância de 5\%. As variáveis independentes utilizadas foram sexo (masculino, feminino), idade ( 0 a 9 e de 10 a 19 anos), os casos confirmados foram as variáveis independentes para as covariáveis (óbito, IAD e razão de leito de UTI), o principal desfecho analisado foi o óbito para os cálculos com prevalência, razão de leitos de UTI e IAD. Além desses, a razão de leitos de UTI foi independente para IAD e prevalência. 
A pesquisa está em conformidade com a Resolução no 510 de sete de abril de 2016, do Conselho Nacional de Saúde por utilizar informações de acesso público sob domínio compartilhado e não precisou da aprovação do Comitê de Ética em Pesquisa.

\section{Resultados}

Até 21 de agosto de 2020, foram confirmados 74.705 casos de Covid-19 em crianças e adolescentes da região Nordeste do Brasil. O primeiro caso foi registrado no estado da Bahia, em 6 de março de 2020. A seguir, tem-se a Tabela 1 com os dados de sexo, faixa etária, número de confirmados e óbitos em crianças e adolescentes.

Tabela 1 - Frequência absoluta e relativa de casos confirmados e óbitos da Covid-19, Brasil, 2020.

\begin{tabular}{lcccc}
\hline \multicolumn{1}{c}{ Variáveis } & \multicolumn{2}{c}{ Casos } & \multicolumn{2}{c}{ Óbitos } \\
\hline \multicolumn{1}{c}{$\mathbf{n}$} & $\mathbf{\%}$ & $\mathbf{n}$ & $\%$ \\
$\quad$ Masculino & 34.554 & 46,3 & 160 & 54,8 \\
Feminino & 40.061 & 53,7 & 132 & 45,2 \\
Faixa etária & & & & \\
0-9 anos & 27.395 & 36,7 & 175 & 60 \\
10-19 anos & 47.310 & 63,3 & 117 & 40 \\
\hline
\end{tabular}

Do total de casos, há prevalência do sexo feminino em casos confirmados da doença, enquanto com relação ao número de óbitos, o sexo masculino teve índices mais expressivos. Em relação à faixa etária, concentrou-se maior número de casos nos adolescentes, dos quais 117 evoluíram a óbito, assim também como parte considerável da população pediátrica morreu em decorrência da infecção pelo Sars-Cov-2.

Estados como Bahia e Ceará obtiveram os maiores índices de notificação da população infantil e pediátrica perfazendo juntos $60,6 \%$ dos casos da região Nordeste. Por outro lado, o 
Análise de indicadores epidemiológicos de crianças e adolescentes acometidos pela Covid... $\mid 8$

estado de Alagoas foi o que menos notificou no período do estudo. Os indicadores em saúde da Covid-19 são apresentados na Tabela 2.

Tabela 2 - Indicadores da Covid-19 em crianças e adolescentes da região Nordeste, Brasil, 2020.

\begin{tabular}{|c|c|c|c|c|c|c|c|}
\hline Estado & População & $\begin{array}{c}\text { Casos } \\
\text { confirmados }^{*}\end{array}$ & Óbitos* & $\begin{array}{c}\text { Letalidade } \\
\text { (\%) }\end{array}$ & $\begin{array}{c}\text { Prevalência } \\
(100.000 \text { hab.) }\end{array}$ & IAD & $\begin{array}{c}\text { Dias após } \\
1^{\circ} \text { caso }\end{array}$ \\
\hline Bahia & 9.917 .375 & $23.531(33,3)$ & $34(11,7)$ & 0,1 & 257 & 140 & 168 \\
\hline Pernambuco & 2.664 .267 & 831 (1) & $66(22,6)$ & 0,8 & 31 & 5,1 & 162 \\
\hline Ceará & 3.035 .091 & $19.497(25,4)$ & $59(20,2)$ & 0,3 & 642 & 119,6 & 162 \\
\hline Maranhã & 2.716 .327 & $12.920(17)$ & $34(11,6)$ & 0,2 & 475 & 82,2 & 156 \\
\hline Paraíba & 1.305 .858 & $3.709(4,8)$ & $18(6,2)$ & 0,4 & 284 & 23,4 & 158 \\
\hline $\begin{array}{l}\text { Rio Grande } \\
\text { do Norte }\end{array}$ & 1.083 .280 & $404(0,5)$ & $12(4,1)$ & 2,9 & 37 & 2,4 & 163 \\
\hline Alagoas & 1.223 .353 & $133(0,2)$ & $20(6,8)$ & 1,1 & 10 & 0,8 & 166 \\
\hline Piauí & 1.131 .904 & $8.493(11)$ & $11(3,8)$ & 0,1 & 750 & 53,7 & 158 \\
\hline Sergipe & 758.267 & $5.187(6,8)$ & $38(13)$ & 2,1 & 684 & 32,4 & 160 \\
\hline Total & 23.835 .722 & 76.705 & 292 & 8 & 321 & 459,6 & - \\
\hline
\end{tabular}

Notas: *n (\%); 'Índice acumulado diário.

Em relação ao número de óbitos, Pernambuco e Ceará possuem os maiores índices, somando 42,8\% da mortalidade nordestina. A prevalência total foi de 321/100.000 habitantes, destacando-se nos estados do Piauí, Sergipe e Ceará. Quanto à letalidade, destaca-se uma mortalidade maior no Rio Grande do Norte, Sergipe e Alagoas. Em relação ao índice acumulado diário, o estado com mais notificações diárias foi a Bahia, seguido do Ceará e Maranhão.

Os leitos neonatais e pediátricos estão distribuídos na região nordeste conforme a Tabela 3. Os leitos pediátricos e neonatais foram detalhados conforme os dados do CNES que identifica a população internada conforme a faixa etária, os adolescentes que foram internados em UTI adulto não foram computados. Destacam-se os estados da Bahia, Pernambuco e Ceará como as unidades com maior quantidade leitos neonatais e pediátricos. Além disso, o estado da Bahia é aquele com maior capacidade de leitos neonatais, enquanto o Pernambuco possui a maior parte dos leitos pediátricos da região. Os leitos destinados ao SUS para a população de 0 a 19 anos 
9 | Neto JC, Feitosa EMS, Silva KVLG, Oliveira CJ

concentram-se na Bahia, por outro lado, o estado de Sergipe é o que tem menos leitos disponíveis pelos SUS (93) na região Nordeste.

Tabela 3 - Distribuição dos leitos de UTI neonatal e pediátrico na região Nordeste, Brasil, 2020.

\begin{tabular}{|c|c|c|c|c|c|c|c|}
\hline \multirow[b]{2}{*}{ Estado } & \multirow[b]{2}{*}{ População } & \multicolumn{6}{|c|}{ Leitos de UTI } \\
\hline & & $\begin{array}{c}\text { Leitos } \\
\text { neonatais* }\end{array}$ & $\begin{array}{c}\text { Leitos } \\
\text { pediátricos* }\end{array}$ & $\begin{array}{l}\text { Número } \\
\text { do SUS }\end{array}$ & $\begin{array}{l}\text { Habilitados } \\
\text { Covid-19 }\end{array}$ & $\begin{array}{l}\text { Habilitados } \\
\text { Covid- } 19^{*}\end{array}$ & $\begin{array}{c}\text { Razão total de } \\
\text { leitos da } \\
\text { UTI/10.000hab }\end{array}$ \\
\hline Bahia & 9.917 .375 & $324(21,8)$ & $133(17,7)$ & 411 & 655 & 17 & 0,46 \\
\hline Pernambuco & 2.664 .267 & $243(16,3)$ & $168(22,3)$ & 353 & 602 & 15 & 1,54 \\
\hline Ceará & 3.035 .091 & $228(15,3)$ & $127(16,9)$ & 305 & 625 & 21 & 1,17 \\
\hline Maranhão & 2.716 .327 & $180(12,1)$ & $60(8)$ & 223 & 238 & - & 0,88 \\
\hline Paraíba & 1.305 .858 & $90(6,1)$ & $77(10,2)$ & 148 & 196 & - & 1,28 \\
\hline $\begin{array}{l}\text { Rio Grande } \\
\text { do Norte }\end{array}$ & 1.083 .280 & $130(8,7)$ & $53(7)$ & 155 & 242 & 15 & 1,69 \\
\hline Alagoas & 1.223 .353 & $144(9,7)$ & $62(8,2)$ & 181 & 202 & - & 1,68 \\
\hline Piauí & 1.131 .904 & $61(4,1)$ & $51(6,8)$ & 101 & 311 & 10 & 0,99 \\
\hline Sergipe & 758.267 & $87(5,9)$ & $22(2,9)$ & 93 & 74 & - & 1,44 \\
\hline Total & 23.835 .722 & 1.487 & 753 & 1.970 & 3.145 & 78 & 11,13 \\
\hline
\end{tabular}

Notas: *n (\%); Números totais de leitos Covid-19; ${ }^{\star}$ Números totais de leitos Covid-19 neonatais e pediátricos.

Com relação aos leitos de UTI habilitados para Covid-19, os estados da Bahia, Ceará e Pernambuco destacam-se pela disponibilidade das unidades de internação para a população geral. Em relação aos leitos destinados a pacientes neonatos e pediátricos, observa-se melhor distribuição no Ceará, Bahia e Pernambuco, com indisponibilidade dos leitos nos estados do Maranhão, Paraíba, Alagoas e Sergipe. A razão de leitos de UTI por habitantes foi menor que dois em todos os estados. Neste caso, somente o Rio Grande do Norte apresentou melhor organização.

Quanto aos cruzamentos dos casos confirmados neonatais e pediátricos e as variáveis óbito $(\mathrm{p}=0.213)$, prevalência $(\mathrm{p}=0.172)$, índice acumulado diário $(\mathrm{p}=0.286)$ e razão de leitos de UTI ( $\mathrm{p}=0.130)$ não houve significância estatística. A relação óbitos e índice acumulado diário foi de $(p=0.338)$. 
Análise de indicadores epidemiológicos de crianças e adolescentes acometidos pela Covid... | 10

Contudo, houve significância para a relação óbito e prevalência $(\mathrm{p}=0.011)$, para óbitos e a razão de leitos de UTI $(\mathrm{p}=0.001)$, prevalência e índice acumulado diário $(\mathrm{p}=0.014)$, prevalência e razão de leitos de UTI $(\mathrm{p}=0.007)$ e para o IAD e a razão de leitos de UTI $(\mathrm{p}=0.020)$.

\section{Discussão}

Os estudos ecológicos permeiam a detecção de aglomerados em espaço-temporais que indicam a melhor forma de planejamento e gestão de recursos, sejam eles voltados aos problemas reais ou potenciais em saúde ou a eminência/vigência de surtos e epidemias, o que denota uma importante maneira de suporte favorecendo o controle da doença ao implementar medidas efetivas de avaliação. Além disso, a criação de um plano emergencial de contingenciamento e mitigação perpassa pelas realidades socioculturais e geográficas do território que alcançam efetividade por meio destes estudos. ${ }^{17-18}$

Ao considerar a rápida dispersão da Covid-19 em todo o território nacional, o monitoramento epidemiológico no Nordeste mostra uma necessidade em planejar a saúde da população viabilizando as realidades socioeconômicas, ambientais, geográficas e sociais. Isso pode favorecer o acompanhamento da pandemia e a capacidade de saúde no Nordeste, viabilizando informações que possam subsidiar a escolha de melhores estratégias para enfrentar a doença. ${ }^{19}$ Os baixos níveis de IDH atrelados à vulnerabilidade da população denota a dificuldade entre diagnóstico e tratamento de enfermidades, dentre eles a Covid-19, além da fragilidade dos serviços públicos do Brasil e na América Latina. ${ }^{20}$

Em estudo, confirmou-se que o estado do Ceará possui uma relação positiva quanto à associação entre a Covid-19 e o desenvolvimento humano, o que denota uma associação entre a desigualdade na incidência de casos no estado, além de quanto mais próximo da região metropolitana maior seria o coeficiente de incidência daquele lugar. ${ }^{21}$ Desde maio de 2020, quando a região Nordeste tinha $31,2 \%$ dos casos, houve um crescimento exponencial no número 
de infectados, chegando a ser a segunda região com mais casos confirmados no território nacional, ${ }^{19,22}$ o que permaneceu até o período desta pesquisa, corroborando com os dados encontrados.

Crianças são mais sensíveis ao Covid-19 e não há uma significativa diferença entre os sexos, por isso eles podem ser transmissores humanos-humanos fazendo com que pacientes adultos se infectem com o vírus. ${ }^{7}$ Estudo realizado no Rio de Janeiro mostrou uma associação por sexo que revelou um percentual de acometimento no sexo masculino de $47,7 \%$ e $51,4 \%$ ao feminino, o que, mesmo em números gerais, coadunam com os dados desta pesquisa. ${ }^{17}$ Este estudo encontrou prevalência do sexo feminino (53,7\%) entre 10 a 19 anos de um total de 47.310 (63,3\%). Uma pesquisa revelou que em adolescentes de ambos os sexos, têm-se percentual de 336 $(46,1 \%)$ em infecção pela Covid-19, o que corrobora com os dados deste estudo.7

Este estudo revelou que o Ceará e a Bahia possuem o maior número de notificações e casos confirmados para Covid-19 em crianças e adolescentes, contudo, em relação aos óbitos, prevalecem os estados de Pernambuco e Ceará. Esses achados reafirmam o que foi encontrado em pesquisa similar ao evidenciar um crescimento exponencial no número de casos também nesses estados. ${ }^{19}$

Quanto à prevalência dos casos da Covid-19 em crianças e adolescentes, o estudo encontrou 321/100.000 habitantes. Isso pode estar relacionado ao número acumulado de casos de neonatos, crianças e adolescentes que são infectados pela Covid-19, mas assintomáticos. Mesmo com a divulgação internacional da infecção pelo Sars-Cov-2 e da emergente necessidade de contenção da doença, existe uma lacuna no conhecimento em relação às implicações clínicas em crianças, sendo relacionados apenas os casos sintomáticos. ${ }^{23}$

A letalidade, estimada em $8 \%$, esteve concentrada nos estados do Rio Grande do Norte, Sergipe e Alagoas. Estudo que caracterizou a síndrome inflamatória multissistêmica em crianças, identificou que há repercussões negativas para os infantes com desfechos 
Análise de indicadores epidemiológicos de crianças e adolescentes acometidos pela Covid... $\mid 12$

desfavoráveis se associados à Covid-19.,7 A letalidade encontrada está acima daquela estimada pela Organização Mundial de Saúde (OMS) de 3,4\%. Contudo, nos estados brasileiros, um estudo nacional identificou falha na prevenção desse agravo onde nenhum dos estados atingiram essa meta. $^{24}$

Nesse sentido, ainda que precoce a definição de caso na clínica pediátrica, as recomendações indicam abordar os menores de 19 anos sem predileção por outras doenças purulentas, sem agravos clínicos quanto à volemia e doenças hipertensivas. Para a população pediátrica, deve-se incluir exames específicos de imagem e monitorização dos parâmetros vitais por meio do eletrocardiograma e elevação de troponina (um importante biomarcador), além de marcadores da resposta inflamatória e evidência de sintomas gastrointestinais. O principal exame, contudo, é o RT-PCR positivo para Covid-19.3,24

Um estudo que revelou alta prevalência do número de casos de Covid-19 em adolescentes até 19 anos demostrou importante susceptibilidade desse público em comparação a faixa etárias mais velhas por estarem em contato com as atribuições escolares, seja no ensino secundário ou no ensino médio e até a faculdade. ${ }^{25}$ Os motivos para tal acontecimento não foram bem justificados na pesquisa, mas os dados corroboram com a presente investigação, uma vez que identificou prevalência no público adolescente.

Por isso, ao considerar o alto percentual de assintomáticos, especialmente de crianças e adolescentes, é importante manter o sistema de vigilância por meio das investigações e confirmações laboratoriais a fim de observar os casos omissos e heterogêneos. ${ }^{23} \mathrm{O}$ maior índice acumulado por estados foi o da Bahia, com 140 casos por dia, dentre eles de crianças, adolescentes, adultos e idosos. Isso ressalta a proporção ampliada da transmissão comunitária do vírus, o que em parte pode se explicar pelas proporções continentais do país, diferenças climáticas e diferentes padrões sazonais entre as regiões brasileiras. ${ }^{26}$ 
O Ministério da Saúde do Brasil tem incentivado esforços para atender à recomendação da OMS de testar casos suspeitos para a detecção dos positivos e orientar o isolamento dos casos da doença e de seus contatos domiciliares, a fim de que se reduza a disseminação do vírus e diminua as peculiaridades que o torna diferente principalmente nas faixas de menor idade. ${ }^{27}$

Nove recém-nascidos foram identificados com infecção pela Covid-19 em Wuhan (China) em 2020, sendo a asfixia considerada o principal agravo relacionado à infecção. Em prematuros houve alteração das enzimas miocárdicas sem demais sintomas clínicos, considerando a manifestação da doença como leve ou moderada. Assim, características de uma possível transmissão vertical devem ser investigadas. ${ }^{28}$

Um estudo sobre as características clínicas de uma coorte de 36 crianças com Covid-19 na China descobriu que aproximadamente metade dos casos era assintomática ou representava apenas sintomas respiratórios agudos superiores leves e a outra metade com uma pneumonia leve e, com isso, pode-se observar e constatar que as manifestações da Covid-19 em crianças são muito mais leves que nos adultos. ${ }^{12}$ Outro estudo com uma série de casos em Bérgamo, Itália, acometendo também exclusivamente a faixa etária pediátrica, evidenciou um aumento de 30 vezes na incidência de manifestações clínicas e alterações laboratoriais que se assemelham a uma síndrome conhecida como síndrome de Kawasaki. ${ }^{29}$

Até 21 de agosto de 2020 havia 3.145 leitos de UTI habilitados para Covid-19 na região Nordeste e destes, apenas 78 eram de unidade neonatal/pediátrica. Até 2017 houve uma redução no número de estabelecimentos $(3,7 \%)$ e leitos de internação hospitalar $(8,4 \%)$ por cem mil habitantes. As UTIs cresceram com disponibilidade de 6,8/100 mil habitantes. ${ }^{16}$ Ao comparar dados nacionais, a região Nordeste tem razão de 11,13, número maior do que os leitos de internação comum estimados nacionalmente.

A razão de leitos de UTI estimada na região Nordeste para o internamento da população geral foi de 1,04/10 mil habitantes, com abertura de licitação nacional para alocar mais mil leitos 
Análise de indicadores epidemiológicos de crianças e adolescentes acometidos pela Covid... | 14

em todo território, além de equipamentos de proteção individual ${ }^{19}$ Entretanto, o estudo em tela revela que para neonatos e pediátricos essa estimativa chega a três vezes esse valor.

Os leitos pediátricos com maior razão estão no estado do Rio Grande do Norte com 1,69\% para cada 10.000 pacientes, isso se deve, necessariamente, aos planos de contingenciamento. Os estados elaboraram planos de contingenciamento capazes de classificar em níveis as respostas entre alerta, perigo iminente e emergência em saúde pública com base no risco e o impacto na saúde pública com trabalhos de vigilância, suporte laboratorial, controle de infecção, assistência, gestão de risco e em saúde. ${ }^{30}$

As heterogeneidades dos estados nordestinos evidenciam a insuficiência de leitos, apontam para a necessidade de insumos e concentram-se na precariedade dos diagnósticos oportunos para o tratamento designado. ${ }^{19}$ Além disso, têm-se a necessidade do diagnóstico diferencial e da captação de crianças e adolescentes a fim de diminuir a infecção ao promover a detecção precoce e o tratamento direcionado.

Para um diagnóstico diferencial, deve-se observar a febre prolongada, erupção cutânea, olhos vermelhos, mãos e pés em anasarca, lábios ou língua doloridos ou com dormência e a presença de linfonodo crescido no pescoço, principalmente porque a interleucina 6 dificulta a distinção entre as respostas inflamatórias sistêmicas da Covid-19 e da doença de Kawasaki. ${ }^{6}$ Entretanto, reforça-se a lavagem das mãos, distanciamento social e a diminuição do risco de infecções nosocomiais como os principais meios de prevenção no cuidado aos infantes.

Por limitações, apresenta-se a subnotificação dos estados em decorrência da demora no processo de coleta e resultado do material, bem como da indisponibilidade de recursos humanos para gerenciamento dos sistemas de saúde e atualização dos boletins. A análise do número de leitos de UTI e os aspectos inerentes aos estudos ecológicos como a dificuldade no processo de análise e a identificação dos casos da amostra também constituem deficiências. 
15 | Neto JC, Feitosa EMS, Silva KVLG, Oliveira CJ

\section{Conclusão}

Os indicadores aqui analisados demonstram a diversidade quanto à caracterização dos dados e à evolução dos casos por estado, em decorrência de divergências estruturais e de gestão. A taxa de letalidade foi de $8 \%$ com prevalência de 321/100.000 habitantes. Destaca-se os estados do Piauí e Rio Grande do Norte com alta prevalência e letalidade, respectivamente. Houve razão significativa entre o número de óbitos, prevalência e a razão de leitos de UTI.

O IAD esteve associado à proporção de casos acumulados desde os primeiros acometimentos da doença nos estados, incluindo os casos de adolescentes e crianças. Os indicadores que tiveram associação com os leitos mostraram a deficiência de leitos habilitados para Covid-19 destinados à população até 19 anos.

A insuficiência de leitos hospitalares habilitados para Covid-19 nos estados está associada à precarização dos serviços públicos antes e durante a pandemia e há fatores que correspondem diretamente às taxas de mortalidade observadas. A razão de leitos de UTI acima dos níveis nacionais denota uma resposta positiva quanto à disponibilidade dessas unidades também no setor infantil. Nesse sentido, são necessários esforços para ampliar a vigilância epidemiológica na identificação, diagnóstico e tratamento dessa população.

Reforça-se que os planos de contingenciamento adotados pelos governos estaduais desde o início da pandemia até agora revelam a necessidade de estratégias governamentais voltadas ao público infanto-juvenil. Contudo, ressalta-se que o estudo contribui para o conhecimento acerca do processo epidêmico da Covid-19 em crianças e adolescentes, assim como abre caminhos a análises constantes que possam indicar o comportamento real da doença, além do planejamento de ações efetivas, coerentes e resolutivas.

\section{Referências}

1. World Health Organization (WHO). WHO Coronavirus disease (COVID-19) dashboard [Internet]. 2020 
Análise de indicadores epidemiológicos de crianças e adolescentes acometidos pela Covid... | 16

$\begin{array}{lllll}\text { [cited } & 2020 & \text { Sept } & 19] . & \text { Available }\end{array}$ https://covid19.who.int/?gclid=cj0kcqjw0mb3brcaarisapsngpvnueov_h1jsrkhboiolp8gxkljnxlltslt6lrserhzk enxjod-qtyaaq3zealw_wcb

2. Lucas LS, Alvin A, Porto DM, Silva AG, Pinheiro MIC. Impactos da pandemia de Covid-19 na saúde mental de crianças e adolescentes: orientações do departamento de psiquiatria da infância e adolescência da Associação Brasileira de Psiquiatria. Debates Psiquiatr [Internet]. 2020 [acesso em 2020 set 19]:74-8. Disponível em: https:/www.uniad.org.br/wpcontent/uploads/dlm_uploads/2020/08/c37608_710e91abd4dd4d088000a38df7ad64c7.pdf

3. Viner RM, Whittaker E. Kawasaki-like disease: emerging complication during the Covid-19 pandemic. Lancet. 2020;395(10239):1741-3. doi: https://doi.org/10.1016/S0140-6736(20)31129-6

4. Lu X, Zhang L, Du H, Zhang J, Li YY, Qu J, et al. SARS-CoV-2 infection in children. N Engl J Med. 2020;382(17):1663-5. doi: https://doi.org/10.1056/NEJMc2005073

5. Yonker LM, Neilan AM, Bartsch Y, Patel AB, Regan J, Arya P, et al. Pediatric severe acute respiratory syndrome Coronavirus 2 (SARS-CoV-2): clinical presentation, infectivity, and immune responses. J Pediatr. 2020;227:45-52. doi: https://doi.org/10.1016/j.jpeds.2020.08.037

6. Bertoncelli D, Guidarini M, Greca AD, Ratti C, Falcinella F, Iovane B, et al. COVID19: potential cardiovascular issues in pediatric patients. Acta Biomed [Internet]. 2020 [cited 2020 Sept 19];91(2):177-83. Available from: https://pubmed.ncbi.nlm.nih.gov/32420942/

7. Dong Y, Mo X, Hu Y, Qi X, Jiang F, Jiang Z, et al. Epidemiology of COVID-19 among children in China. Pediatrics. 2020;145(6):1-12. doi: https://doi.org/10.1542/peds.2020-0702

8. Vilelas JMS. The new coronavirus and the risk to children's health. Rev Latinoam Enferm. 2020;28:1-2. doi: https://doi.org/10.1590/1518-8345.0000.3320

9. Götzinger F, Santiago-García B, Noguera-Julián A, Lanaspa M, Lancella L, Carducci FIC, et al. COVID-19 in children and adolescents in Europe: a multinational, multicentre cohort study. Lancet Child Adolesc Health. 2020;4(9):653-61. doi: https://doi.org/10.1016/S2352-4642(20)30177-2

10. Lima CMAO. Information about the new coronavirus disease (COVID-19). Rev Bras Radiol. 2020;53(2):5-6. doi: https://doi.org/10.1590/0100-3984.2020.53.2e1

11. Chih-Cheng L, Yen Hung L, Cheng-Yi W, Ya-Hui W, Shun-Chung H, Muh-Yen Y, et al. Asymptomatic carrier state, acute respiratory disease, and pneumonia due to severe acute respiratory syndrome coronavirus 2 (SARS-CoV-2): facts and myths. J Microbiol Immunol Infect. 2020;53(3):404-12. doi: https://doi.org/10.1016/j.jmii.2020.02.012

12. Safadi MAP. As características intrigantes da COVID-19 em crianças e seu impacto na pandemia. J 
Pediatr (Rio J). 2020;96(3):265-8. doi: https://doi.org/10.1016/j.jped.2020.04.001

13. Pacheco ES, Silva VR, Soares LS. A brief epidemiological analysis of Covid-19 in Piauí, Brazil. Rev Prev Infecç Saúde. 2020;6:1-15. doi: https://doi.org/10.26694/repis.v6i0.10690

14. Caldas RM, Sampaio YSB. Pobreza no Nordeste Brasileiro: uma análise multidimensional. Rev Econ Contemp. 2015;19(1):74-96. doi: https://doi.org/10.1590/198055271914

15. Instituto Brasileiro de Geografia e Estatística (IBGE). IBGE cidades: Brasil: panorama [Internet]. Brasília (DF): 2020 [acesso 2020 set 19]. Disponível em: https://cidades.ibge.gov.br/brasil/panorama

16. Fundação Oswaldo Cruz (FIOCRUZ), Avaliação do Desempenho do Sistema de Saúde (PROADESS). Boletim Informativo do PROADESS: monitoramento da assistência hospitalar no Brasil (2009-2017) [Internet]. Rio de Janeiro: Fundação Oswaldo Cruz; 2019 [acesso 2020 set 19]. 26p. Disponível em: https://www.proadess.icict.fiocruz.br/Boletim_4_PROADESS_Monitoramento\%20da\%20assistencia\%20h ospitalar_errata_1403.pdf

17. Cavalcante JR, Abreu AJL. COVID-19 no município do Rio de Janeiro: análise espacial da ocorrência dos primeiros casos e óbitos confirmados. Epidemiol Serv Saúde. 2020;29(3):1-10. doi: https://doi.org/10.5123/s1679-49742020000300007

18. Boulos MNK, Geraghty EM. Geographical tracking and mapping of coronavirus disease COVID19/severe acute respiratory syndrome coronavirus 2 (SARS-CoV-2) epidemic and associated events around the world: how 21st century GIS technologies are supporting the global fight against outbreaks and epidemics. Int J Health Geogr. 2020;19:8. doi: https://doi.org/10.1186/s12942-020-00202-8

19. Marinelli NP, Albuquerque LPA, Sousa IDB, Batista FMA, Mascarenhas MDM, Rodrigues MTP. Evolução de indicadores e capacidade de atendimento no início da epidemia de COVID-19 no Nordeste do Brasil, 2020. Epidemiol Serv Saúde. 2020;29(3):e2020226. doi: https://doi.org/10.5123/s167949742020000300008

20. Rodriguez-Morales AJ, Gallego V, Escalera-Antezana JP, Mendéz CA, Zambrano LI, Franco-Paredes C, et al. COVID-19 in Latin America: the implications of the first confirmed case in Brazil. Travel Med Infect Dis. 2020;35:101613. doi: https://doi.org/10.1016/j.tmaid.2020.101613

21. Maciel JAC, Castro-Silva II, Farias MR. Análise inicial da correlação espacial entre a incidência de COVID-19 e o desenvolvimento humano nos municípios do estado do Ceará no Brasil. Rev Bras Epidemiol. 2020;23(1):e200057. doi: https://doi.org/10.1590/1980-549720200057

22. Ministério da saúde (BR). Coronavírus Brasil: painel Coronavírus [Internet]. 2020 [acesso em 2020 ago 21]. Disponível em: https://covid.saude.gov.br/

23. Iser BPM, Sliva I, Raymundo VT, Poleto MB, Schuelter-Trevisol F, Bobinski F. Definição de caso 
Análise de indicadores epidemiológicos de crianças e adolescentes acometidos pela Covid... $\mid 18$

suspeito da COVID-19: uma revisão narrativa dos sinais e sintomas mais frequentes entre os casos confirmados. Epidemiol Serv Saúde. 2020;29(3):e2019354. doi: https://doi.org/10.5123/s167949742020000300018

24. Organização Pan-Americana da Saúde (OPAS). Organização Mundial da Saúde (OMS). Síndrome inflamatória multissistêmica em crianças e adolescentes com a doença causada pelo novo coronavírus (COVID-19) [Internet]. Brasília (DF): Organização Pan-Americana da Saúde; 2020 [acesso em 2020 set 19]. Disponível em: https://iris.paho.org/handle/10665.2/52176

25. Rumain BT, Schneiderman M, Geliebter A. Prevalence of COVID-19 in adolescents and youth compared with older adults in states experiencing surges. MedRxiv. 2020;10.20.20215541. doi: https://doi.org/10.1101/2020.10.20.20215541

26. Ministério da Saúde (BR). Nota técnica GVIMS/GGTES/Anvisa № 07/2020 - atualizada em 05/08/2020 [Internet]. Brasília (DF): Ministério da Saúde; 2020 [acesso em 2020 ago 26]. Disponível em: https://www20.anvisa.gov.br/segurancadopaciente/index.php/alertas/item/nota-tecnica-gvims-ggtesanvisa-n-07-2021

27. Ministério da Saúde (BR). Saúde amplia testes para profissionais de saúde e segurança [Internet]. 2020. Brasília (DF): Ministério da Saúde; 2020 [acesso em 2020 ago 26]. Disponível em: https://antigo.saude.gov.br/noticias/agencia-saude/46596-saude-amplia-testes-para-profissionais-desaude-e-seguranca

28. Chen H, Guo J, Wang C, Luo F, Yu X, Zhang W, et al. Clinical characteristics and intrauterine vertical transmission potential of COVID-19 infection in nine pregnant women: a retrospective review of medical records. Lancet. 2020;395(10226):809-15. doi: https://doi.org/10.1016/S0140-6736(20)30360-3

29. Verdoni L, Mazza A, Gervasoni A, Martelli L, Ruggeri M, Ciuffreda M, et al. An outbreak of severe Kawasaki-like disease at the Italian epicentre of the SARS-CoV-2 epidemic: an observational cohort study. Lancet. 2020;395(10239):1771-8. doi: https://doi.org/10.1016/S0140-6736(20)31103-X

30. Ministério da Saúde (BR). Fundação Oswaldo Cruz (FIOCRUZ). Plano de contingência da Fiocruz diante da pandemia da doença pelo SARS-CoV-2 (Covid-19) [Internet]. Brasília (DF): Fundação Oswaldo Cruz; 2020 [acesso em 2020 ago 26]. Disponível em: https://portal.fiocruz.br/sites/portal.fiocruz.br/files/documentos/plano_de_contingencia_corona_final_202 0-03-13_v1.pdf

Editora Científica Chefe: Cristiane Cardoso de Paula

Editora Científica: Tânia Solange Bosi de Souza Magnago

Fomento / Agradecimento: O presente estudo não possui financiamento. 
19 | Neto JC, Feitosa EMS, Silva KVLG, Oliveira CJ

\section{Autor Correspondente}

João Cruz Neto

E-mail:enfjcncruz@gmail.com

Endereço: Coronel Antônio Luiz, 1161 - Pimenta, Crato, Ceará, Brasil

CEP: 63105-000

\section{Contribuições de Autoria}

\section{1 - João Cruz Neto}

Concepção, análise e interpretação dos dados, redação, revisão crítica do conteúdo e aprovação da versão final do manuscrito.

\section{2 - Emanuel Messias Silva Feitosa}

Análise e interpretação dos dados, redação, revisão crítica do conteúdo e aprovação da versão final do manuscrito.

\section{3 - Kely Vanessa Leite Gomes da Silva}

Revisão crítica e intelectual do conteúdo e aprovação da versão final do manuscrito.

\section{4 - Célida Juliana de Oliveira}

Revisão crítica e intelectual do conteúdo e aprovação da versão final do manuscrito.

\section{Conflitos de Interesse}

Os autores declaram não haver conflitos de interesse.

\section{Como citar este artigo}

Neto JC, Feitosa EMS, Silva KVLG, Oliveira CJ. Análise de indicadores epidemiológicos de crianças e adolescentes acometidos pela Covid-19 no Nordeste do Brasil. Rev. Enferm. UFSM. 2021 [Acesso em: Anos Mês Dia]; vol.11 e:19 1-19. DOI: https://doi.org/10.5902/2179769263043 